\title{
EFEITOS DE MODALIDADE DE LÍNGUA: AS LÍNGUAS DE SINAIS
}

\section{Ronice Muller de Quadros}

\begin{abstract}
"The most important thing I want to stress is that ASL is a language. Of course, it seems completely different from familiar languages such as English, Russian, and Japanese. Its means of transmission is not through the speaker's vocal tract creating acoustic signals that are detected by the addressee's ears. Rather, the speaker's gestures create signals detected by the addressee's visual system. (...) The peripheral system is different, but the inner workings are exactly the same."
\end{abstract}

(JACKENDOFF, 1994:83)

\section{RESUMO}

As línguas de sinais que são visuais-espaciais oferecem um campo de análise que se refere aos possíveis efeitos que a diferença na modalidade pode implicar para as teorias lingüísticas e para as análises discursivas. Neste artigo, vamos nos deter a discutir os efeitos de modalidade na perspectiva teórica apresentando uma revisão dos estudos clássicos das línguas de sinais, bem como dos estudos que buscam compreender as especificidades dessas línguas.

\section{PALAVRAS-CHAVE}

Línguas de sinais; Efeitos de modalidade; Modalidade visual-espacial

\section{LANGUAGE MODALITY EFFECTS: THE SIGN LANGUAGES}

\begin{abstract}
Sign languages that are visuo-spatial languages offer an area of analyses that refer to possible effects in which the difference in the modality can have implication to the linguistic theories and to the discourse analyses. In this paper, we will discuss the modality effects in the theoretical perspective presenting a revision of the classical studies of sign languages, as well as the studies that look for understanding the specificity of the languages.
\end{abstract}

\section{KEYWORDS}

Sign languages; Modality effects; Visual-spatial modality 
As línguas de sinais que são visuais-espaciais oferecem um campo de análise que se refere aos possíveis efeitos que a diferença na modalidade pode implicar para as teorias lingüísticas e para as análises discursivas. Neste artigo, vamos nos deter a discutir os efeitos de modalidade na perspectiva teórica apresentando uma revisão dos estudos clássicos das línguas de sinais, bem como dos estudos que buscam compreender as especificidades dessas línguas.

\section{O QUE É DIFERENTE E O QUE É IGUAL?}

Na sua grande maioria, os lingüistas têm se ocupado em identificar o que é comum entre as línguas de sinais e as línguas faladas. Parte-se dos referenciais já propostos para as línguas faladas e os universais lingüísticos que também foram estabelecidos a partir de estudos com várias línguas faladas e propõem-se análises das línguas de sinais.

Esta linha investigativa justifica-se também, uma vez que na década de 60 havia um movimento intenso no sentido de “provar” que as línguas de sinais eram, de fato, línguas naturais.

Atualmente, não há dúvidas em relação ao estatuto lingüístico das línguas de sinais. Assim, principalmente a partir da década de 90, iniciaram-se investigações com o intuito de identificar não apenas o que era “igual”, mas também o que era “diferente” com o objetivo de enriquecer as teorias lingüísticas atuais.

A pergunta que antes era "Como a lingüística se aplica às línguas de sinais ou dá conta das línguas de sinais?” passou a ser “Como as línguas de sinais podem contribuir para os estudos lingüísticos?”

A mudança, aparentemente sutil, abre novos caminhos investigativos no campo da lingüística buscando explicações para o que é diferente entre estas modalidades de língua, inclusive com o exercício de olhar as línguas de sinais a partir delas mesmas enquanto línguas visuais-espaciais.

\section{OS ESTUDOS CLÁSSICOS DAS LÍNGUAS DE SINAIS}

Os estudos dos últimos 40 anos revelam similaridades profundas entre as línguas faladas e línguas sinalizadas tanto no nível da estrutura da frase, quanto no nível do processamento lingüístico e, também, quanto à aquisição da linguagem. A seguir, 
apresentamos uma síntese de alguns estudos considerados clássicos em relação às línguas de sinais: Stokoe (1960) apresentou a primeira análise lingüística da língua de sinais americana com evidências de que um sinal era resultado de combinações de unidades menores: a configuração da mão, o local de articulação e o movimento. Stokoe apresenta uma análise com base na simultaneidade, ou seja, as unidades são combinadas simultaneamente para a produção do sinal.

Battison (1974) inclui a orientação da mão como parâmetro na fonologia das línguas de sinais com base na existência de pares mínimos em sinais que apresentam mudança de significado apenas na produção de distintas orientações da palma da mão.

Battinson (1978) apresenta as restrições fonológicas na produção de diferentes tipos de sinais que restringem a complexidade dos sinais (por exemplo, a condição de simetria e a condição de dominância com sinais produzidos com duas mãos).

Klima \& Bellugi (1979) evidenciaram que o conjunto possível das unidades que constituíam os sinais poderia variar de língua para língua, mas de forma bastante restrita. Também apresentam uma análise detalhada do sistema derivacional e flexional da língua de sinais americana.

Baker (1976) e Liddell (1980) apresentam uma descrição dos adjetivos, dos advérbios e de expressões faciais que coocorrem com os sinais manuais de forma sistemática. Tais autores argumentam que tais expressões faciais são lexicais, uma vez que são altamente restringidas ao contexto dessas classes de palavras.

Liddell (1984) apresenta uma análise da seqüencialidade dos sinais através da representação interna da estrutura do sinal e as relações de dependências entre os seus segmentos. Além disso, aponta que os sinais coocorrem com expressões faciais e movimentos do corpo que são interpretados como advérbios ou informações gramaticais adicionais.

Fischer (1973); Klima \& Bellugi (1979); Fischer \& Gough (1978); Supalla (1982) e Padden (1983) analisam as diferentes classes de verbos na língua de sinais americana: os verbos simples (plain verbs) que aceitam apenas a flexão de aspecto; os verbos de movimento (verbs of motion) que não flexionam para pessoa, número e nem aspecto, mas apresentam uma morfologia complexa e os verbos com flexão (inflecting verbs) que flexionam para pessoa, número e aspecto. Estes autores apresentam análises da sintaxe espacial.

Liddell e Johnson (1989) e Sandler (1989) desenvolvem modelos que não são apenas simultâneos, mas apresentam uma seqüência estrutural, por exemplo, um sinal pode ter duas CM ou L em uma seqüência. Esta proposta tornou possível uma análise do morfema de (C) ETD - Educação Temática Digital, Campinas, v.7, n.2, p.168-178, jun. 2006 - ISSN: 1676-2592. 170 
concordância consistindo de traços de locação como afixo independente que é ajuntado ao verbo não especificado para a locação.

Lillo-Martin (1991) apresenta uma análise da sintaxe da língua de sinais com respaldo no processo de aquisição da linguagem em crianças surdas filhas de pais surdos. A autora evidencia que a língua de sinais americana é analisável segundo os princípios e parâmetros propostos pela teoria gerativa.

Neidle, Kegl, MacLaughlin, Bahan \& Lee (2000) apresentam a sintaxe da língua de sinais americana. Os autores apresentam marcações manuais e não manuais como expressões de traços sintáticos abstratos. Desenvolvem uma análise da estrutura da língua de sinais com base na teoria gerativa.

Ferreira-Brito (1995) apresenta uma breve descrição lingüística da língua de sinais brasileira incluindo, principalmente, alguns aspectos fonológicos e morfológicos. A autora menciona que o estudo de uma língua em uma modalidade visual-espacial pode afetar as teorias lingüísticas quanto aos preceitos teóricos, quanto à gramática ao se rever a noção de arbitrariedade, a noção de linearidade e a noção do que seja central em uma determinada língua (p.11-12).

Karnopp $(1994,1999)$ apresenta um estudo mais voltado para a representação fonológica da língua de sinais brasileira. Analisa, inclusive, as implicações de processos fonológicos na aquisição da língua de sinais brasileira.

Quadros (1995, 1999) desenvolve uma análise da estrutura da língua de sinais brasileira. Sua proposta decorre da classificação dos verbos nesta língua que apresentam ou não concordância.

Quadros e Karnopp (2004) apresentam uma descrição lingüística da língua de sinais brasileira nos níveis fonológico, morfológico e sintático.

\section{OS ESTUDOS EM BUSCA DE EFEITOS DA MODALIDADE}

Alguns estudos têm se ocupado no sentido de identificar e analisar os efeitos da modalidade da língua na estrutura lingüística. As evidências têm sido identificadas como conseqüências das diferenças nos níveis de interface articulatório-perceptual. Algumas investigações têm ainda levantado algumas hipóteses quanto à possíveis diferenças no nível da interface conceptual implicando em uma semântica enriquecida em função de propriedades 
visuais-espaciais. A seguir, apresentamos alguns dos estudos que representam a identificação dos efeitos da modalidade das línguas de sinais:

Padden (1988) apresenta a discussão sobre o papel do espaço nas línguas de sinais. Espaço na língua de sinais não é apenas uma entidade semântica, um espaço mental, mas um dos elementos que faz parte de uma unidade lexical. A pergunta formulada pelos pesquisadores diante dos pontos espaciais estabelecidos no discurso das línguas de sinais é de como tais pontos podem ser representados na gramática. Onde estes pontos espaciais (pronomes) são especificados no léxico?

Lillo-Martin e Klima (1986) propõem uma análise para este problema: há apenas uma entrada para pronome com locação não específica (uma variável), mas com a interpretação determinada através do discurso.

Liddell $(1990,1995)$ sugere que os pontos no espaço devem ser descritos como entidades mentais (pictóricas). Segundo sua análise, tais entidades não podem fazer parte do sistema lingüístico, pois envolvem espaços reais contendo uma representação mental do objeto/referência em si. Assim, não há necessidade de definir o locus fonológica e morfologicamente. Além disso, a concordância verbal deixa de existir enquanto concordância do ponto de vista lingüístico.

Rathmann e Mathur (2002) analisam a proposta de Liddell e mostram que o problema se apresenta considerando os níveis de variação fonética dos locus em línguas de sinais, sendo eles formal e de determinação de fronteiras. No primeiro caso, se se estabelecesse um ponto no espaço para JOÃO no lado esquerdo, se tenta voltar ao mesmo ponto ao referir JOÃO durante o discurso. No segundo caso, um ponto diferente do ponto estabelecido para JOÃO pode ter um significado diferente. Uma vez que há correspondência entre o ponto e o referente, cada locus deve ser listado no léxico. Apesar dos locus de não-primeira pessoas fazerem parte de um conjunto que apresenta "ligação" dentro do discurso, o critério do léxico que determina listabilidade não é observado. Assim, o problema de infinitude está relacionado com a listabilidade. Os autores destacam também evidências lingüísticas (e psicolingüísticas) de que concordância existe na língua de sinais americana. Apesar da existência destes classificadores, parece que o sistema lingüístico é ordenado de forma linear em algum nível que obviamente não é trivial.

Lillo-Martin (2002) justifica a existência de concordância nas línguas de sinais como elemento gramatical a partir de vários aspectos lingüísticos, entre eles, a autora menciona os seguintes: as formas para primeira pessoa e não-primeira pessoa são diferentes; a presença de (c) ETD - Educação Temática Digital, Campinas, v.7, n.2, p.168-178, jun. 2006 - ISSN: 1676-2592. 172 
marcação de número nos verbos apresenta múltiplas formas em diferentes línguas de sinais; a existência de auxiliar em algumas línguas de sinais que expressam a relação sujeito-verboobjeto nas construções com verbos que não marcam concordância. No entanto, Lillo-Martin destaca que há um tipo de construção, os verbos chamados de verbos manuais e/ou verbos classificadores, que parece romper com todas as regras na língua de sinais em todos os níveis de análise (sintático, morfológico e fonológico), uma vez que apresenta um comportamento completamente incomum considerando as análises clássicas de um item lexical.

Liddell (2000) tende a analisar tais construções como expressões de ordem não sintática. Esse viés é retomado nas suas análises mais atuais excluindo por completo uma análise de ordem sintática nos termos analisados até então considerando a teoria lingüística e os estudos das línguas em geral. Sua proposta segue um rumo alternativo. Sua versão, na verdade, resulta de uma atenção especial às diferenças, uma vez que assim poder-se-ia estar adentrando nos limites da teoria lingüística. Liddell $(1990,1995)$ considera que os pontos estabelecidos no espaço que são incorporados pelos verbos no que vem se chamando de concordância, não podem ser analisados morfologicamente, uma vez que tais pontos são indeterminados. A partir de suas análises, ele conclui que não há concordância verbal na língua de sinais americana. Para o autor, o que acontece é uma indicação de natureza gestual combinada com elementos de ordem lingüística dos sinais.

Lillo-Martin (2002) e Quadros (2002) apresentam evidências quanto ao status da concordância na língua de sinais brasileira. As autoras apresentam exemplos para ilustrar que não há uma ordenação caótica nas sentenças incluindo os verbos manuais. Isso indica que, apesar das características essencialmente visuais e espaciais, há restrições quanto à ordenação dos constituintes na estrutura. Tais construções seguem o mesmo padrão: todas ocupam a posição final da sentença. Com os classificadores, o predicado complexo inteiro que inclui o verbo ocupa esta posição. Todos os exemplos estão ou associado com a marcação não-manual de concordância ou com a marcação não manual de tópico. Em termos estruturais, a posição final também é ocupada pelo foco que usualmente está associada com o movimento da cabeça, mas têm-se exemplos de que há restrições de tal posição ser ocupada por argumentos oracionais. Uma hipótese possível seria considerar estas construções manuais tendo relação em alguma instância com as construções de foco, mas tais argumentos oracionais serem considerados pela sintaxe nucleares, uma vez que morfologicamente apresentam características de um único sinal. Assim, a sintaxe sendo cega a informação semântica oracional, a estrutura seria derivada de qualquer forma apresentando a devida interpretação na (c) ETD - Educação Temática Digital, Campinas, v.7, n.2, p.168-178, jun. 2006 - ISSN: 1676-2592. 173 
interface que do ponto de vista fonológico apresentaria uma interpretação equivalente a um único sinal que pode ser analisado em unidades menores (cf. SUPPALA, 1982, 1986).

Considerando esses estudos, parece haver efeitos de modalidade que se refletem na própria estrutura da língua. Segundo Liddell (1995), a informação sobre a relação entre a atividade e o objeto envolvido é claramente expressada de forma espacial num sentido pictórico. Um exemplo seria o seguinte:

WOMAN PIE PUT-IN-OVEN

A Mulher colocou a torta no forno.

(Liddell, 1980:89-91)

BALL JOHN SWING-A-BAT

John bateu na bola com um taco.

FENCE CAT SLEEP

O gato dormiu na cerca sentado.

(Liddell, 1980:91-100)

Interessantemente, tais exemplos na língua de sinais brasileira apresentam estes verbos manuais/classificadores na posição final:

JOÃO PAREDE PINTAR-ROLO

João pinta a parede com o rolo.

JOÃO CARRO [CL(carro)-BATER-POSTE - ]cl

O João estava de carro e deu uma batidinha no poste.

JOÃO MARIA [CL(pessoa)-CRUZAR-UM-PELO-OUTRO]

O João cruzou pela Maria.

CENTRO PESSOAS [CL(pessoas)-CRUZANDO-ENTRE-SI

No centro, várias pessoas cruzam entre si.

Ao que tudo indica, as derivações visuais-espaciais seguem a mesma lógica das derivações orais-auditivas, no sentido de observar restrições na organização sintática que delimitam as possibilidades existentes na derivação de sentenças. No entanto, as observações de Liddell são pertinentes, em especial, quanto à organização morfológica das palavras classificadoras, apesar de haver argumentos favoráveis a uma análise nos padrões clássicos (Suppala, 1982, 1986). Lillo-Martin (2002) apresenta a partir dessas considerações a seguinte questão: as línguas de sinais podem oferecer alguma informação nova quanto ao nível de interface articulatório-perceptual? 
Nesse sentido, cabe considerar o estabelecimento de pontos no espaço. Do ponto de vista de Liddell, tais pontos não podem ser analisados como representações gramaticais, mas sim pictóricas. De fato, tais pontos não seguem os padrões de análise morfológico clássicos, no entanto, as evidências sintáticas acomodam as análises dentro da perspectiva da teoria lingüística.

Aqui surge ainda outra questão, as informações gramaticais atreladas às marcas nãomanuais que também apresentam um caminho de possibilidades de contribuições para o entendimento das interfaces. Rathmann e Mathur, acomodando a versão de Liddell, propõem que as marcações chamadas neste trabalho como 'manuais' (ou gestuais por Rathmann e Mathur, ou ainda representações espaciais mentais pictóricas por Liddell) podem ser classificadas como concordância no sentido sintático, mas apresentar repercussões no nível articulatório-perceptual.

Muitas pesquisas sobre a estrutura das línguas de sinais têm considerado tais questões, mas ainda tem-se muito a ser investigado. Por um lado, existe uma preocupação em relação aos efeitos das diferenças na modalidade fazendo com que os estudos das línguas de sinais sejam extremamente relevantes. Por outro lado, as similaridades encontradas entre as línguas faladas e as línguas sinalizadas parecem indicar a existência de propriedades do sistema lingüístico que transcendem a modalidade das línguas. Nesse sentido, o estudo das línguas de sinais tem apresentado elementos significativos para a confirmação dos princípios que regem as línguas humanas.

(...) sign languages resemble spoken languages in all major aspects, showing that there truly are universals of language despite differences in the modality in which the language is performed. (FROMKIN; RODMAN, 1993)

\section{REFERÊNCIAS:}

AARONS, D. Aspects of the syntax of American Sign Language. Ph.D. Dissertation, Boston University, Boston, MA. 1994.

BAHAN, B. Non-manual realization of agreement in American Sign Language. Ph.D. Dissertation, Boston University, Boston, MA. 1996.

BOUCHARD, D. Sign Languages \& Language Universals: The status of Order \& Position in Grammar. Sign Language Studies. 91, 1996, p.101-160. 
EMMOREY, k. Repetition Priming with Aspect and Agreement Morphology in American Sign Language. Journal of Psycholinguistic Sign Language. v.20. n.5. 1991. p. 365-388.

FERREIRA-BRITO, L. Por uma gramática das línguas de sinais. Rio de Janeiro: Tempo Brasileiro/UFRJ, 1995.

FISCHER, S. The Head Parameter In ASL. SLR'87 Papers from The Fourth International Symposium on Sign Language Research. Lappeenranta, Finland July 15 - 19, 1987. W.H. Edmondson \& F. Karlsson (eds). Volume 10. SIGNUM - Verlag. Hamburg. 1990. p.75-85.

FISCHER, S. Verb Inflections in American Sign Language and Their Acquisition by the Deaf Child. Paper presented at The Winter Meeting of the Linguistic Society of America. [s.l] [s.n.]. 1973.

FROMKIN, V. \& RODMAN, R. An Introduction to Language. Forth Worth: 5. ed., Harcourt Brace Jovanovich College, 1993.

KARNOPP, L. B. Aquisição do Parâmetro Configuração de Mão dos Sinais da LIBRAS: Estudo sobre quatro crianças surdas filhas de pais surdos. Dissertação (Mestrado) - Instituto de Letras e Artes. Pontifícia Universidade Católica do Rio Grande do Sul, Porto Alegre, 1994.

KARNOPP, L. B. Aquisição fonológica na Língua Brasileira de Sinais: Estudo longitudinal de uma criança surda. Tese (Doutorado) - Pontifícia Universidade Católica do Rio Grande do Sul, Porto Alegre. 1999.

LIDDELL, S. Four Functions of a Locus: Reexaming the Structure of Space. In ASL. In Sign Language Research - Theorical Issues. Gallaudet University Press. Washington. 1990. p. 176-200.

LIDDELL, S. Think and believe: sequentiality. In ASL. Language. 60:372-99. 1984.

LIDDELL, S. American Sign Language Syntax. Mouton Publisher. The Hague. 1980.

LILLO-MARTIN, D. One syntax or two? Sign Language and Syntactic Theory. In Glot International. (to appear)

LILLO-MARTIN, D. Where are all the modality effects? In MEIER, R. P.; CORNIER, K A; QUINTO, D. G. (eds.) Modality and Structure in Signed Language and Spoken Language. Cambridge: Cambridge University Press. 2002.

LILLO-MARTIN, D. C. Universal Grammar and American Sign Language. London : Kluwer Academic Publishers. 1991.

LILLO-MARTIN, D. C. Studies of American Sign Language Syntax and the Principles and Parameters of Universal Grammar. In SLR'87 Papers from The Fourth International Symposium on Sign Language Research. Lappeenranta, Finland July 15 - 19, 1987. v.10. SIGNUM - Verlag. Hamburg. 1990. p.86-93. 
LILLO-MARTIN, D. C. Parameter setting: evidence from use, acquisition, and breakdown in American Sign Language. Doctoral Dissertation. University of California, San Diego. University Microfilms International, Ann Arbor, Michigan. 1986.

MACLAUGHLIN, D. The Structure of Determiner Phrases: Evidence from American Sign Language. Doctoral Dissertation. Boston University. 1997.

MATSUOKA, K. Verb Raising in American Sign Language. Lingua. 103:127-149. 1997.

MEIER, R. A cross-linguistic perspective on the acquisition of inflection morphology in American Sign Language. University of California, San Diego and The Salk Institute for Biological Studies. April. 1980.

PADDEN, C. Interaction of Morphology and Syntax in ASL. Doctoral Dissertation. University of California, San Diego. 1983.

PADDEN, C. Grammatical theory and signed languages. In NEWMEYER, F. J. (Org) Linguistics: The Cambridge Survey .New York: Cambridge University Press. 1988. p.250265.

PADDEN, C. The Relation Between Space and Grammar in ASL Verb Morphology. Sign Language Research - Theorethical Issues. Gallaudet University Press. Washington. 1990. p.118-132.

PETRONIO, K. A focus position in ASL. MIT Working Papers in Linguistics. 14:211-225. Department of Linguistics and Philosophy of MIT. 1991.

PETRONIO, K. Clause Structure in ASL. Ph.D. Dissertation. University of Washington. 1993.

PETRONIO, K. \& LILLO-MARTIN, D. WH Movement and the Spec of CP: Evidence from American Sign Language. Language. Volume 73. Number 1. 1997. p.18-57

PETITTO, L. On the Autonomy of Language and Gesture: Evidence from the Acquisition of Personal Pronouns in American Sign Language. Cognition, vol. 27. 1987. p.1-52.

QUADROS, R. M. A gramática da língua de sinais brasileira. Trabalho apresentado por ocasião da Conferência da ANPOLL. Gramado. 2002 (oral).

QUADROS, R. M. de. Phrase Structure of Brazilian Sign Language. Tese de Doutorado. PUC/RS. Porto Alegre. 1999.

QUADROS, R. M. de. As categorias vazias pronominais: uma análise alternativa com base na língua de sinais brasileira e reflexos no processo de aquisição. Dissertação de Mestrado. PUCRS. Porto Alegre. 1995.

QUADROS, R. M. de. Educação de surdos:a aquisição da linguagem. Artes Médicas. Porto Alegre. 1997. 
RATHMANN, C. and MATHUR, G. Is verb agreement the same cross-modally? In . MEIER, R. P.; CORNIER; K. A.; QUINTO, D. G. (EDS.) Modality and Structure in Signed Language and Spoken Language. Cambridge: Cambridge University Press. 2002.

SHEPARD-KEGL, J. Locative relations in American Sign Language Word Formation, Syntax, and Discourse. Ph.D. Dissertation. MIT. 1985.

SMITH, W. Evidence for Auxiliaries in Taiwan Sign Language. SLR'87 Papers from The Fourth International Symposium on Sign Language Research. Lappeenranta, Finland July 15 - 19, 1987. v.10. SIGNUM - Verlag. Hamburg. 1990. p.211-228.

STOKOE, W. Sign and Culture: A Reader for Students of American Sign Language. Maryland: Linstok Press, 1960.

SUPALLA, T. Structure and Acquisition of Verbs of Motion and Location in American Sign Language. Ph.D. Dissertation, University of California, San Diego. 1982.

SUPALLA, T. The classifier system in ASL. In CRAIG, C. (ORG.). Noun classes and categorization: Typological studies in language. Philadelphia: John Benjamin Publishing Co. 1986. 